\title{
Ecocardiografia no diagnóstico de reticulopericardite traumática bovina
}

\section{Echocardiography in the diagnosis of bovine traumatic reticulopericarditis}

\author{
Bárbara Barbi de Freitas ${ }^{1 *}$, Julio Pereira dos Santos ${ }^{1}$, João Henrique Perotta ${ }^{2}$, Nicoly Nayana Marcom², Mariana \\ Cocco $^{3}$, Thais Gislon da Silva ${ }^{1}$, Juliana Sperotto Brum ${ }^{1}$, Marlos Gonçalves Sousa ${ }^{1}$, Ivan Roque de Barros Filho ${ }^{1}$ \\ ${ }^{1}$ Programa de Pós-Graduação em Ciências Veterinárias, Universidade Federal do Paraná (UFPR), Curitiba PR, Brasil \\ 2 Departamento de Medicina Veterinária, Universidade Federal do Paraná (UFPR), Curitiba, PR, Brasil \\ ${ }^{3}$ Hospital Veterinário, Universidade Federal do Paraná (UFPR), Curitiba, PR, Brasil
}

\section{Resumo}

A reticulopericardite traumática bovina é uma doença caracterizada pelo processo inflamatório resultante da perfuração do retículo e do pericárdio por objeto pontiagudo ingerido. 0 animal tem sinais de dor como abatimento, odontoprise, arqueamento e respiração predominantemente abdominal. Outros sinais podem ocorrer de acordo com as estruturas envolvidas. No caso do pericárdio, pode haver sinais de insuficiência cardíaca congestiva, taquicardia, abafamento das bulhas cardíacas, dispneia, edema e distensão da veia jugular. 0 objetivo deste trabalho é relatar um caso de reticulopericardite traumática bovina, atendido no Hospital Veterinário da UFPR, com enfoque nos achados ecocardiográficos. Por meio dos sinais clínicos e exames complementares, foi possível conduzir a suspeita diagnóstica para reticulopericardite traumática. Para confirmação desta suspeita foi realizado exame ecocardiográfico, onde foi possível visualizar espessamento pericárdico, bem como efusão pleural e pericárdica, confirmando o diagnóstico. Apesar de a ecocardiografia ser um método não invasivo, ela ainda não é amplamente utilizada na clínica de bovinos, e no presente trabalho mostrou-se eficaz e de grande importância para definição do diagnóstico e prognóstico do animal.

Palavras-chave: Pericardite. Diagnóstico. Efusão. Disfunção diastólica.

\begin{abstract}
Traumatic reticulopericarditis in cattle is a disease characterized by inflammation of the reticulum and pericardium perforation by a sharp ingested object. The animal shows signs of pain, such as indulgence, bruxism, bowing, and predominantly abdominal breathing. Other signs may occur according to the structures involved, when the pericardium may show signs of congestive heart failure, tachycardia, muffled heart sounds, dyspnea, edema, and distension of the jugular vein. The objective of this paper is to report a case of traumatic
\end{abstract}


reticulopericarditis in a cow treated at the Veterinary Hospital of Federal University of Paraná, Curitiba, Brazil, focusing on the echocardiographic findings. Based on the clinical signs and laboratory findings, it was possible to conduct the presumptive diagnosis of traumatic reticulopericarditis. In order to validate presumption, an echocardiographic examination was performed, in which it was possible to visualize the pericardial thickening, as well as the pericardial and pleural effusions. These observations confirmed the diagnosis. In addition, although echocardiography is a non-invasive method, it is still not widely used in cattle. In the case studied, the examination proved effective and of great value for the diagnosis and prognosis of the condition of the animal.

Keywords: Pericarditis. Diagnosis. Effusion. Diastolic dysfunction.

\section{Introdução}

A reticulopericardite traumática bovina (RPT) é uma doença caracterizada pelo processo inflamatório resultante da perfuração do retículo e do pericárdio por objeto perfurante ingerido (Radostits et al., 2002; Anteneh e Ramswamy, 2015). Além do retículo, o corpo estranho também pode perfurar o coração, resultando em miocardite (Dirksen et al., 2002). 0 diagnóstico é feito por meio do histórico, exame físico geral, específico, e exames complementares como a ferroscopia, laparoscopia e ultrassonografia (Dirksen et al., 1993, 2002). 0 exame ecocardiográfico pode ser uma importante alternativa para diagnóstico em casos de reticulopericardite(Braun, 2009).

A enfermidade tem importância econômica devido às perdas produtivas e descarte de animais. Alguns trabalhos têm sido realizados a fim de verificar a ocorrência de reticulopericardite traumática nos bovinos. Em Minas Gerais foi observada a presença de 62 casos de pericardites, num total de 18.877 animais abatidos, com uma prevalência de 0,33\% (Oliveira et al., 2013). No estudo de Lucena et al. (2010), no período de 1964 a 2008, a RPT representou 19,3\% dos distúrbios fatais causados por agentes físicos em bovinos.0 objetivo deste estudo é relatar um caso de RPT bovina com enfoque nos achados ecocardiográficos.

\section{Relato de caso}

Foi atendida no Setor de Grandes Animais do Hospital de Veterinário da Universidade Federal do Paraná uma vaca, mestiça, com três anos de idade, pesando $310 \mathrm{Kg}$, com histórico de parto eutócico há 40 dias, apresentando hiporexia e aparente cansaço. 0 animal recebeu as vacinas contra brucelose e febre aftosa, foi medicado anteriormente na propriedade com tilosina (15 $\mathrm{mg} / \mathrm{Kg} \mathrm{SID} / 3$ dias), imidocarb $(1,0 \mathrm{mg} / \mathrm{Kg}$ em dose única) e flunixin meglumine $(2,2 \mathrm{mg} / \mathrm{Kg}$ SID $/ 2$ dias $)$.

No hospital veterinário, durante o exame físico geral, constatou-se hipotermia $\left(36,9^{\circ} \mathrm{C}\right)$, frequência respiratória de $40 \mathrm{mpm}$, movimentos ruminais fracos ( $2 \mathrm{mov} / 3 \mathrm{~min}$ ) e frequência cardíaca de $116 \mathrm{bpm}$. A mucosa vaginal e as conjuntivas palpebrais estavam ligeiramente pálidas. Apresentava odontoprise, dificuldade de locomoção, edema na região do peito. Na avaliação do sistema cardiovascular havia hipofonese cardíaca e taquicardia. Além disso, apresentava aumento da área cardíaca à percussão, ingurgitamento das veias jugulares e prova do garrote positiva. As provas do bastão e pinçamento da cernelha foram consideradas negativas e ferroscopia positiva (Dirksen et al., 1998). Os valores de hemograma e análises bioquímicas da paciente estão contidos na Tabela 1 .

Para o exame de ecocardiografia o animal foi contido em posição quadrupedal em tronco. Após depilação local, foi realizada a abordagem entre o $3^{\circ}$ e $4^{\circ}$ espaço intercostal (EIC) do lado direito e entre o 4o e 5o EIC do lado esquerdo.Foi empregado aparelho de ultrassonografia (EsaoteMyLab ${ }^{\text {TM }} 30$ Gold VET) transdutor setorial de frequência variável de 4 - 2 $\mathrm{mHz}$, este posicionado 5 a 10 centímetros acima da cartilagem xifoide.

Iniciando pela janela paraesternal esquerda foi possível identificar conteúdo anecóico heterogêneo com pontos ecogênicos em suspensão. Somado a isso havia estruturas finas de bordos lisos, imagem que sugeriu presença de líquido livre na cavidade pleural. Na mesma janela, a partir da identificação dos ventrículos e átrios, foi percebida no espaço pericárdico, próximo à parede livre do ventrículo esquerdo (VE), a presença de conteúdo ecogênico homogêneo medindo $2,8 \mathrm{~cm}$ no eixo curto x $16,4 \mathrm{~cm}$ no eixo longo. Ainda do lado esquerdo, o pericárdio 
visceral apresentou espessura de $17,2 \mathrm{~mm}$ enquanto o pericárdio parietal apresentou $6,9 \mathrm{~mm}$. Foram visualizados pontos hiperecóicos, não formadores de sombra acústica entremeados entre o conteúdo, indicando possível presença de gás (Figura 1).

A avaliação quantitativa foi realizada no corte transversal na janela paraesternal direita, posicionando a linha de pesquisa na altura dos músculos papilares do VE. Esta janela foi usada para mensurar o diâmetro do ventrículo esquerdo e para medir a espessura da parede livre e do septo interventricular tanto em sístole quanto em diástole (Figura 2, Tabela 2).

Reticulopericardite foi o diagnóstico estabelecido nos sinais clínicos, exames laboratoriais e no exame ultrassonográfico. Devido a tratamento pregresso com antimicrobiano e anti-inflamatório ineficientes, estado clínico crítico, comprometimento importante do bem-estar da paciente, além do prognóstico ruim, o proprietário optou pela eutanásia.

Tabela 1 - Valores de hemograma, bioquímica clínica da paciente e respectivos valores de referência

\begin{tabular}{|c|c|c|c|c|c|}
\hline \multicolumn{2}{|c|}{ Hemograma } & \multirow{2}{*}{$\begin{array}{c}\text { Valor de referência* } \\
5,0-10,0 \times 10^{6} / \mu \mathrm{L}\end{array}$} & \multicolumn{2}{|c|}{ Análises bioquímicas } & \multirow{2}{*}{$\begin{array}{c}\text { Valor de referência* } \\
3,3-3,5 \mathrm{~g} / \mathrm{dL}\end{array}$} \\
\hline Hemácias & $6,62 \times 10^{6} / \mu \mathrm{L}$ & & Albumina & $2,2 \mathrm{~g} / \mathrm{dL}$ & \\
\hline Hematócrito & $30 \%$ & $24-46(\%)$ & AST & $89,5 \mathrm{UI} / \mathrm{L}$ & 20,0 - 34,0 UI/L \\
\hline Hemoglobina & $8,6 \mathrm{~g} / \mathrm{dL}$ & $8,0-15,0 \mathrm{~g} / \mathrm{dL}$ & Creatinina & $0,8 \mathrm{mg} / \mathrm{dL}$ & $1,0-2,0 \mathrm{mg} / \mathrm{dL}$ \\
\hline Leucócitos totais & $24.200 / \mu \mathrm{L}$ & $4.000-12.000 / \mu \mathrm{L}$ & GGT & $62,4 \mathrm{UI} / \mathrm{L}$ & 6,1 - 17,4 UI/L \\
\hline N. Segmentados & $22.022(91 \%)$ & $600-4000(15-45 \%)$ & Uréia & $28,0 \mathrm{mg} / \mathrm{dL}$ & $42,8-64,2, \mathrm{mg} / \mathrm{dL}$ \\
\hline Linfócito & $1210(5 \%)$ & $2500-7500(45-75 \%)$ & Fibrinogênio plasmático & $1,3 \mathrm{~g} / \mathrm{dL}$ & $0,3-0,7 \mathrm{~g} / \mathrm{dL}$ \\
\hline Eosinófilo & $726(3 \%)$ & $0-2400(2-20 \%)$ & & & \\
\hline Monócito & $242(1 \%)$ & $25-840(2-7 \%)$ & & & \\
\hline
\end{tabular}

Nota: *Lopes et al., 2007.

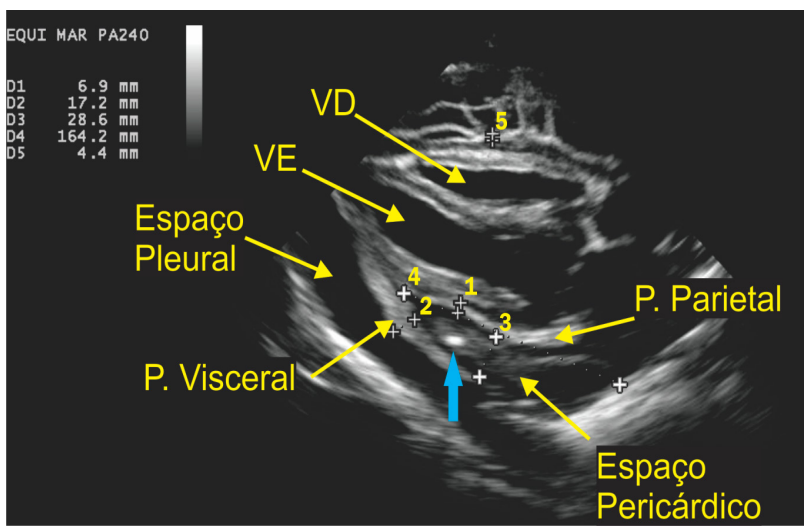

Nota: P. Parietal - Pericárdio parietal. P. Visceral - Pericárdio Visceral. VD ventrículo direito.VE - ventrículo esquerdo. Medida dos cursores: 1-Espessura do pericárdio visceral lado esquerdo; 2 - Espessura do pericárdio visceral; 3 - Dimensão transversal do espaço pericárdico; 4 - Dimensão longitudinal do espaço pericárdico; 5 - Espessura do pericárdio parietal do lado direito.

Figura 1 - Janela paraesternal esquerda, corte longitudinal demonstrando as quatro câmaras cardíacas e a lesão no pericárdio. Nota-se a presença de gás formando um ponto hiperecóico (seta azul).

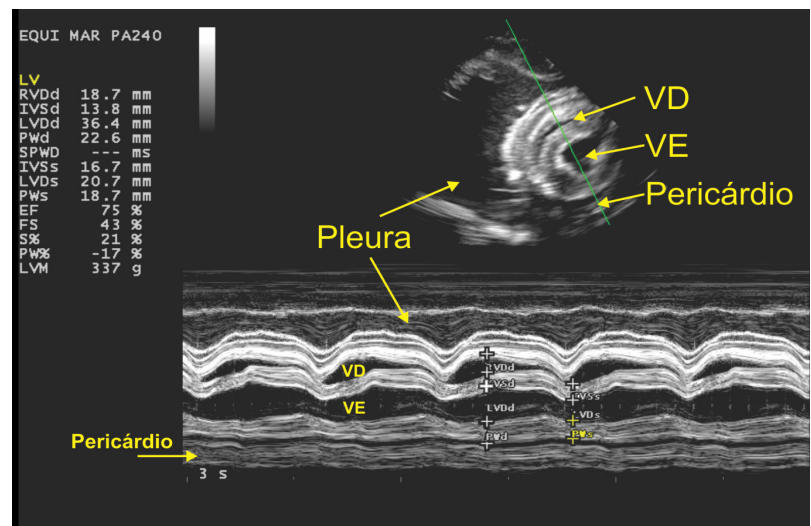

Nota: PVD - ventrículo direito. VE - ventrículo esquerdo.

Figura 2 - Janela paraesternal direita. Na parte superior é exibida a imagem bidimensional, onde se pode observar o corte transversal dos ventrículos na altura dos músculos papilares. Na parte inferior tem-se o gráfico do modo-M, onde é possivel observar o espaço pleural e 0 espaço pericárdico, ambos com conteúdo ecogênico. É possível notar a hipocinesia da parede livre do VE, a qual, durante a sístole, apresentou espessura menor quando comparada à espessura em diástole. 
Tabela 2 - Medidas ecocardiográficas da vaca avaliada e valores de referência da literatura

\begin{tabular}{l|ccc}
\hline & Vaca & Jersey $^{*}$ & Holstein-Fresian* $^{*}$ \\
\hline Septo (d) mm & 13,8 & $20,0 \pm 4,0$ & $22,0 \pm 5,1$ \\
Ventrículo esq. (d) mm & 36,4 & $77,0 \pm 7,0$ & $80,7 \pm 1,0$ \\
Parede livre VE (d) mm & 22,6 & $12,0 \pm 3,0$ & $15,0 \pm 4,0$ \\
Septo (s) mm & 16,7 & $36,0 \pm 5,0$ & $34,0 \pm 5,0$ \\
Ventrículo esq. (s) mm & 20,7 & $42,0 \pm 5,3$ & $42,0 \pm 8,0$ \\
Parede livre VE (s) mm & 18,7 & $15,0 \pm 5,0$ & $14,0 \pm 5,0$ \\
FEc\% & 43,0 & $44.7 \pm 8,3$ & $46,5 \pm 9,5$ \\
Frequência cardíaca & 116,0 & $71,0 \pm 6,0$ & $72,3 \pm 4,5$ \\
\hline
\end{tabular}

Nota: FEc\% - Fração de encurtamento,(d) - diástole,(s) - sístole. * Parâmetros segundo Halowell et al., 2007.

Nos achados necroscópicos foi observado aproximadamente $2 \mathrm{~L}$ de líquido translúcido amarelado na cavidade abdominal (hidroperitônio). À abertura do retículo foram observados múltiplos objetos metálicos filiformes e rígidos, variando aproximadamente de 3 a $5 \mathrm{~cm}$ de comprimento e 0,2 a $0,4 \mathrm{~cm}$ de espessura, transfixando a mucosa. Líquido semelhante ao do abdômen foi observado em maior quantidade, aproximadamente $4 \mathrm{~L}$, na cavidade torácica (hidrotórax). 0 saco pericárdico apresentava a parede marcadamente espessada. À abertura, observou-se que o espaço pericárdico estava distendido e obstruído por grande quantidade de material denso, amarelado, formando longos filamentos (fibrina), ocasionando aderências entre os folhetos parietal e visceral, caracterizando lesão crônica.

\section{Discussão}

A reticulopericardite traumática é a cardiopatia mais relatada em bovinos (Buczinski et al., 2010a), sendo caracterizada como um processo posterior à reticuloperitonite (Radostits et al., 2002). No presente caso, o histórico do animal de anorexia e perda de peso é compatível com o descrito por outros autores (Sojka et al., 1990; Roth et al., 1991;. A presença de corpo estranho perfurante no retículo, com lesão à mucosa, torna a alimentação um processo doloroso para o animal.
Dispneia e taquipneia são causadas pela dor decorrente do movimento dos pulmões e do diafragma lesionado pelo objeto perfurante. 0 aumento da taxa respiratória funciona como mecanismo compensatório (Braun et al., 2018).

A paciente apresentou resultado negativo nas provas do bastão e pinçamento da cernelha. Os bovinos diferem na resposta à dor em relação a outras espécies; suas formas de demonstrar dor podem estar associadas a outros comportamentos (Anderson e Muir, 2005). Por se tratar de um processo crônico, pode haver diminuição da sensibilidade dolorosa (Dirksen et al., 2002). A ferroscopia é útil na demonstração de corpo estranho metálico na região (Ollhoff e Birgel, 2012).

A constatação de leucocitose por neutrofilia e linfopenia, bem como a hiperfibrinogenemia, representa a resposta inflamatória ao processo infeccioso associado à perfuração do retículo e diafragma (Reedy et al., 2014; Braun et al., 2018). Também foi constatado aumento da atividade das enzimas hepáticas aspartato aminotransferase (AST) e gama glutamiltransferase (GGT), indicando comprometimento hepático, que é secundário ao aumento da pressão hidrostática venosa, levando à congestão hepática (Braun et al., 2007; Ghanem, 2010; Imran et al., 2011).

0 pericárdio é formado por dois folhetos, parietal e visceral. No processo ocorre a perfuração das estruturas e a inoculação de microorganismo, seguida pela deposição de fibrina entre os folhetos pericárdicos, que produz um ruído proveniente do atrito entre os as duas partes durante o ciclo cardíaco (Athar et al., 2012). Com a evolução do quadro, há formação de efusão no espaço entre os dois folhetos. Na auscultação, o resultado é o abafamento dos sons cardíacos e hipofonese (Braun et al., 2007). A presença de bactérias anaeróbicas pode levar à formação de conteúdo gasoso, semelhante ao caso relatado.

0 espessamento da parede pericárdica, com presença de material denso fibrinoso, deveu-se ao processo inflamatório crônico, fazendo com que a região afetada fosse recoberta com substancial quantidade de fibrina (Athar et al., 2012). Com a evolução do processo inflamatório ocorreu o desenvolvimento da efusão pericárdica (Imran et al., 2011).0 pericárdio de animais hígidos não deve ser visível no exame ecocardiográfico (Athar 
et al., 2012), contudo o caso relatado apresentou espessamento confirmado na necropsia. A pericardite também pode evoluir para pericardite constritiva, que é prejudicial para a função diastólica (Buczinski et al., 2010a).

A produção de altas quantidades de fibrina, bem como a formação de aderências entre os folhetos do pericárdio, possivelmente ocasionou um processo constritivo, reduzindo a capacidade diastólica, que por fim levou o ventrículo esquerdo a apresentar diâmetro diastólico menor que as referências (Hallowell et al., 2007).

As lesões miocárdicas ocasionadas pelos objetos pontiagudos somadas à restrição de relaxamento miocárdico levaram à redução na movimentação da parede livre do ventrículo esquerdo, a qual apresentou espessura menor durante a sístole se comparada à espessura em diástole, visível na avaliação em modo-M (Tabela 2, Figura 2). Concluise, portanto, que a parede livre estava hipocinética na região próxima ao acúmulo de conteúdo no pericárdio. A fração de encurtamento (FEc\%), que é a diferença do diâmetro diastólico e sistólico do VE, permaneceu dentro dos níveis de normalidade, contudo esse resultado deve ser interpretado com parcimônia devido à influência da pré e da póscarga neste resultado.

0 organismo lança mão de mecanismos neuroendócrinos para aumentar a frequência e elevar o débito cardíaco. Contudo, no momento em que esses mecanismos tornam-se insuficientes, o resultado é a congestão venosa, que promove os sinais de insuficiência cardíaca congestiva (ICC) no paciente. Em bovinos é mais prevalente a ICC de origem direita (Braun, 2009; Buczinski et al., 2010b).

Os achados de necropsia puderam ser previamente visualizados por meio da ecocardiografia, tanto o espessamento do pericárdio, como a presença de efusão pericárdica e pleural.

\section{Conclusão}

Os achados clínicos e epidemiológicos na RPT dos bovinos são fundamentais no diagnóstico, mas em algumas situações o clínico veterinário pode necessitar de exames complementares que o auxiliem. A utilização de exame ecocardiográfico realizado por operador treinado permitiu a visualização de espessamento dos folhetos pericárdicos, presença e caracterização de efusões pericárdica e pleural, bem como avaliação dos diâmetros dos ventrículos e da dinâmica de suas paredes, evidenciando o comprometimento do órgão. Com o animal contido, a obtenção das imagens foi de fácil realização, contudo essa prática do estudo ecocardiográfico ainda é pouco difundida na rotina da clínica de bovinos. Apesar de ser um método não invasivo, a ecocardiografia ainda não é amplamente utilizada na clínica de bovinos, e no presente trabalho mostrou-se eficaz e de grande importância para a definição do diagnóstico e prognóstico do animal.

\section{Referências}

Anderson DE, Muir WW. Pain management in cattle. Vet Clin North Am Food Anim Pract. 2005;21(3):623-35, v-vi.

Anteneh M, Ramswamy V. Hardware disease in bovine (Review article). Acad J Anim Dis. 2015; 4(3):146-59.

Athar H, Parrah JD, Moulvi BA, Singh M, Dedmari FH. Pericarditis in bovines - A review. Int J Adv Vet Sci Tech. 2012;1(1):19-27.

Braun U, Lejeune B, Schweizer G, Puorger M, Ehrensperger F. Clinical findings in 28 cattle with traumatic pericarditis. Vet Rec. 2007;161(16):558-63.

Braun U. Traumatic pericarditis in cattle: clinical, radiographic and ultrasonographic findings. Vet J. 2009;182(2):176-86.

Braun U, Warislohner S, Torgerson P, Nuss K, Gerspach C. Clinical and laboratory findings in 503 cattle with traumaticreticuloperitonitis. BMC Vet Res. 2018;14(1):66.

Buczinski S, Francoz D, Fecteau G, DiFruscia R. Heart disease in cattle with clinical signs of heart failure: 59 cases. Can Vet J. 2010a; 51(10):1123-9.

Buczinski S, Rezakhani A, Boerboom D. Heart disease in catlle: diagnosis, therapeutic approaches and prognosis. Vet J. 2010b;184(3):258-63. 
Dirksen G, Gründer HD, Stöber M. Rosenberber - Exame Clínico dos Bovinos. 3 ed. Rio de Janeiro: Guanabara Koogan; 1993. 419 p.

Dirksen G, Gründer HD, Baumgartner W, Braun U. Innere Medizin und Chirurgie des Rindes. 4 ed. Berlin: Parey Buchverlag; 2002. 1325 p.

Ghanem MM. A comparitive study on traumatic reticuloperitonitis and traumatic pericarditis in egyptian cattle. Turk J Vet Anim Sci. 2010; 34(2):143-53.

Hallowell GD, Potter TJ, Bowen IM. Methods and normal values for echocardiography in adult dairy cattle. J Vet Cardiol. 2007;9(2):91-8.

Imran S, Tyagi SP, Kumar A, Kumar A, Sharma S. Ultrasonographic application in the diagnosis and prognosis of pericarditis in cows. Vet Med Int. 2011;2011:974785.

Lopes STA, Biondo AW, Santos AP. Manual de Patologia Clínica Veterinária. 3 ed. Santa Maria: UFSM, Departamento de Clínica de Pequenos Animais; 2007. $107 \mathrm{p}$.
Lucena RB, Pierezan F, Kommers GD, Irigoyen LF, Fighera RA, Barros CSL. Doenças de bovinos no Sul do Brasil: 6.706 casos. Pesq Vet Bras. 2010;30(5):428-34.

Oliveira HC, Silva LC, Cunha Filho LFC, Santana EHW, Bogado ALG, Negri Filho LC, et al. Ocorrência de reticulo pericardite traumática em bovinos de abate, na região de Araguari-MG. Rev Bras Hig Sanid Anim. 2013;7(2):192-202.

Ollhoff RD, Birgel Jr EH. Detecção de corpos estranhos metálicos por meio de ferroscopia em rebanho bovino de leite de alta produção. Arq Inst Biol. 2012;79(3):415-7.

Radostits OM, Gay CC, Blood DC, Hinchcliff KW. Clínica Veterinária: Um Tratado de doenças dos bovinos, ovinos, suínos, caprinos e equinos. 9 ed. Rio de Janeiro: Guanabara Koogan; 2002. 1772 p.

Roth L, King JM. Traumatic reticulitis in cattle: A review of 60 fatal cases. J Vet Diagn Invest. 1991;3(1):52-4.

Sojka JE, White MR, Widmer WR, Vanalstine WG. An unusual case of traumatic perdicardittis in a cow. J Vet Diagn Invest. 1990;2(2):139-42. 